\title{
TuPG07
}

\section{Correlation Analysis of Fracture Intensity Descriptors with Different Dimensionality in a Geomechanics-constrained 3D Fracture Network}

W. Zhu' ${ }^{1 *}$, B. Yalcin ${ }^{1}$, S. Khirevich ${ }^{1}$, T. Patzek ${ }^{1}$

${ }^{1}$ King Abdullah University of Science and Technology

\section{Summary}

3D intensity parameters of fracture networks cannot be measured directly and are usually correlated with the lower dimensionality intensity parameters, such as P21, P10. A comprehensive correlation analysis between lower dimensionality measures, P10, P20, P21, I2D (total number of intersections per unit area) and higher dimensionality ones, P30, P32, I3D (total number of intersections per unit volume) are investigated. We also correlate small cube samples and underlying fracture networks that represent cores or tunnels. The fracture networks are constrained by geomechanics principles and outcrop data to make them geologically meaningful. We show that orientation of fracture samples impacts correlations between the 2D and 3D parameters and samples parallel to the principal stresses yield better correlations. 3D intensity parameters, P30, I3D, and P32 can be predicted from 2D or small cube samples. However, 1D intensity P10 doesn't have a strong correlation with 3D intensity parameters. The size of cube samples should be larger than 10 percent of the original size to capture main structural information. Furthermore, the minimum number of samples to reach a good correlation from $2 \mathrm{D}$ and cube samples are 20 and 60 respectively. 


\section{Introduction}

The knowledge of fracture sizes, shapes and connections is necessary to describe "intensity" of a fracture network. Dershowitz (1992) defined the fracture intensity measures in different dimensions, denoted as $P_{i j}$, where $i$ refers to the dimension of the sample and $j$ refers to the dimension of the measure. For example, $P_{10}$ is the number of fractures per unit length and $P_{21}$ is the length of fracture traces per unit area, etc. Available measures are usually $1 \mathrm{D}$ and 2D, e.g., $P_{10}$ from image $\operatorname{logs}, P_{20}$ and $P_{21}$ from outcrop studies, etc. However, the real fracture networks are 3D and their intensity cannot be measured directly. The 3D intensity parameters are crucial in many engineering applications, and it is necessary to find correlations between the lower dimensionality measures and the higher dimensionality ones, if these correlations exist. Dershowitz et al. (2000) found that the 1D intensity measure $P_{10}$ and the 2D measure $P_{21}$ are often linearly correlated with the 3D intensity measure $P_{32}$. Wang (2005) found that $P_{10}$ and $P_{32}$ have a linear correlation under certain assumptions about the distributions of fracture lengths, positions and orientations. Comprehensive analysis of fracture intensity parameters in different dimensions is rarely possible, mostly because of insufficient data. Since real fracture networks are complex and their geometry and topology are poorly known, it is impossible to model these networks exactly. Therefore, stochastic fracture networks are the only practical alternative (Zhu et al., 2018). With our algorithms, we can generate arbitrary stochastic fracture networks in 2D and 3D. These networks may follow any distribution of fracture lengths, orientations, and positions of fracture centers. Our networks can be investigated systematically for the correlations between the $1 \mathrm{D}$ and $2 \mathrm{D}$ intensity measures, and the 3D measures. In this work, we generate a typical fracture network constrained by geomechanics and outcrop data, which includes four types of joints. Then we conduct the scanline surveys and crosssection surveys to investigate correlations between lower dimensionality measures, $P_{10}, P_{20}, P_{21}$, and the higher dimensionality ones, $P_{30}$ and $P_{32}$. Since percolation in a fracture network depends on fracture intersections, we also include the intersection density, which is defined as the total number of intersections per unit area in $2 \mathrm{D}, I_{2 D}$, or the total number of intersections per unit volume in $3 \mathrm{D}, I_{3 D}$. Finally, we investigate the correlations between small cube samples and the original 3D fracture networks. These samples correspond to cores or tunnels.

\section{Correlation analysis of different intensity parameters in geomechanics-constrained fracture net- works}

In this paper, we constrain fracture networks with simple geomechanics principles and outcrop characteristics to make them more geologically meaningful. To this effect, we have introduced four types of joints, sketched in Fig. 1a. Type 1 joints are in blue and type 2 joints are in green. These are tension joints that are, respectively, approximately parallel and perpendicular to the hinge line. The Type 3 conjugate shear joints (actually microfaults) are in red. They have dihedral angles equal to $60^{\circ}$ and their angle bisectors are parallel to the maximum principal stress $\sigma_{1}$. Type 4 shear joints (microfaults) are in cyan. They have random strikes and dips because of the local anisotropy. The existence of random shear joints brings more complexity and uncertainties to the network. The system size in this paper is $100^{3}$ of arbitrary units. The orientations of the maximum and minimum principal stress $\sigma_{1}, \sigma_{3}$ are north-south and east-west, respectively. The distributions of fracture lengths, strike angles, dip angles and the positions of fracture centers are listed in Table. 1 . The minimum length of fracture $L_{\min }$ used in a power law distribution is 3 initially. When a spanning cluster is formed in the system, $L_{\min }$ reduces to 1.5 and the probabilities for type 3 and 4 joints increase to 0.43 and 0.53 . After a spanning cluster emerges, the pore pressure propagates across the system, makes the local stress field more complex and easier for shear failures. Therefore, it generates smaller secondary joints and more random shear joints. Fig. $\mathbf{1 b}$ is an outcrop map cited from Holland et al. (2009), and the joint intensity is far beyond the percolation threshold.Therefore, the termination criterion in our model is a number of total fractures randomly distributed in $[45000,55000]$, which is about 10 times larger than the percolation requirement. Fig. 1c is a cross-section map of our fracture network. Fig. $\mathbf{~ b}$ and $\mathrm{c}$ are not identical, but they share many common characteristics, like preferential fracture orientations, different fracture sets, etc. We generate 100 stochastic fracture networks and calculate the $P_{30}, P_{32}$ and $I_{3 D}$ for each one of them. After that, we take 100 scanline samples and 100 cross-section samples, respectively, in each 3D fracture network, calculate the corresponding parameters, $P_{10}, P_{20}, P_{21}$ and $I_{2 D}$, and average them. The 


\section{P $\equiv T R$ R O L $=U M M 2019$ GEOSTATISTICS}

Table 1 Distributions of each type of joints.

\begin{tabular}{|c|c|c|c|c|c|}
\hline Type of joints & Probability $^{a}$ & Center position & Strike & Dip & Length \\
\hline 1 & 0.02 & Uniform $^{b}$ & $\begin{array}{c}\text { von Mises-Fisher } \\
\left(\mu=90^{\circ}, \kappa=300\right)\end{array}$ & $90^{\circ}$ & $2 \mathrm{~L}$ \\
\hline 2 & 0.02 & Uniform & $\begin{array}{l}\text { von Mises-Fisher } \\
\left(\mu=0^{\circ}, \kappa=300\right)\end{array}$ & $90^{\circ}$ & $\begin{array}{c}\text { Power-lawe } \\
\left(L_{\max }=L, a=3\right)\end{array}$ \\
\hline 3 & 0.72 & Uniform & ${ }^{d} \mathrm{~N} 60^{\circ} \mathrm{E}, \mathrm{S} 60^{\circ} \mathrm{E}$ & $90^{\circ}$ & $\begin{array}{c}\text { Power-law } \\
\left(L_{\max }=L, a=2.5\right)\end{array}$ \\
\hline 4 & 0.24 & Fractal $^{c}$ & $\begin{array}{l}\text { Uniform } \\
([0,2 \pi])\end{array}$ & $\begin{array}{l}\text { Uniform } \\
([0,2 \pi])\end{array}$ & $\begin{array}{c}\text { Power-law } \\
\left(L_{\max }=L, a=3\right)\end{array}$ \\
\hline
\end{tabular}

a probability of generation.

${ }^{b}$ a uniform spatial distribution.

${ }^{c}$ a fractal spatial density distribution and the fractal dimension is 2.5 in this research.

${ }^{d}$ the dihedral angles equal to $60^{\circ}$ and angle bisectors are parallel to $\sigma_{1}$.

${ }^{e} L_{\max }$ is the maximum length of the fracture; a is the exponent of the power-law distribution.

orientations of the scanlines and cutting-planes are either uniformly distributed between $[0,2 \pi]$ or are orthogonal to principal stresses. In each 3D fracture network, we take 100 small cube samples. The sample sizes vary between 1 and 35 percent of the system size. We calculate the intensity parameters in the cube samples, denoted as $P_{30 s}, P_{32 s}$ and $I_{3 D s}$. Fig.2 is a sketch map of the different samples from the fracture network.

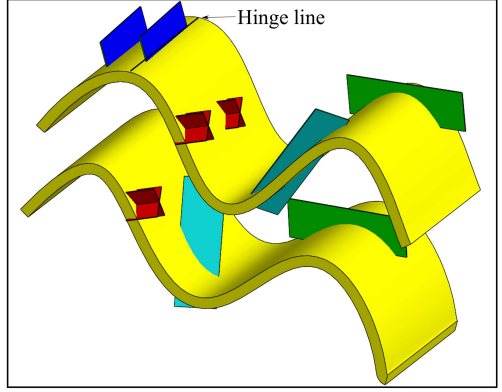

$\mathrm{a}$

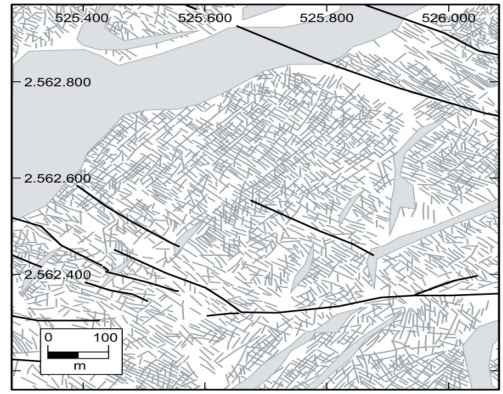

b

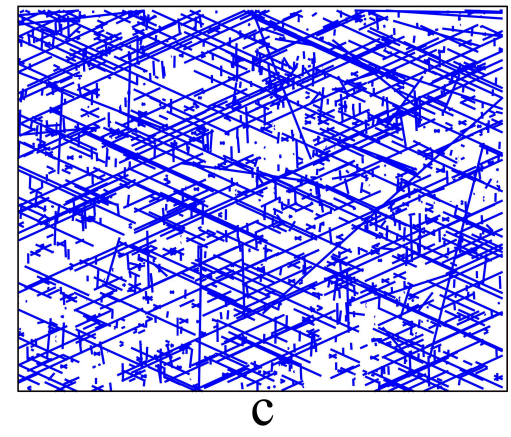

C

Figure 1 a: A sketch map to illustrate four types of joints in a fold structure; $\boldsymbol{b}$ : An outcrop map from Holland et al. (2009); c: A cross-section map of our fracture network.

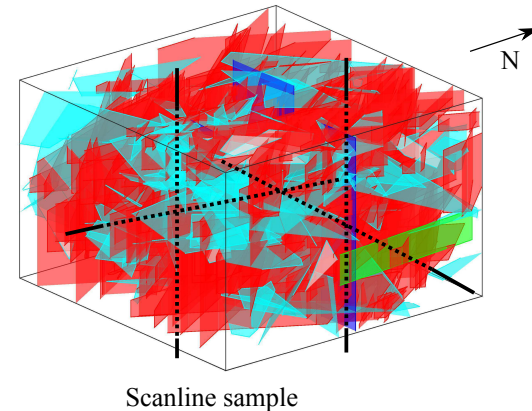

Scanline sample

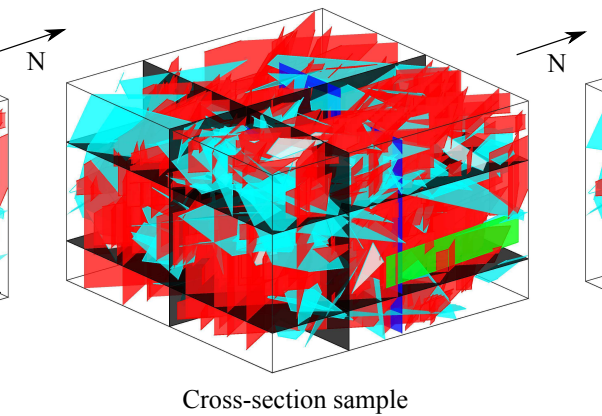

Cross-section sample

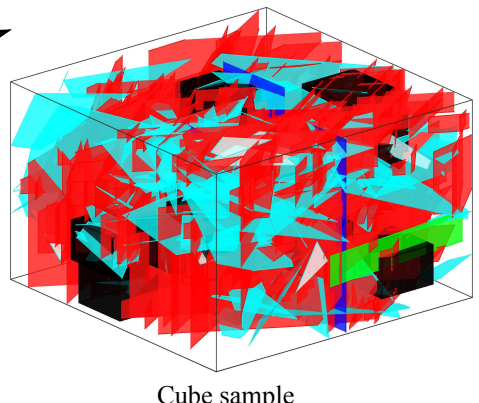

Cube sample

Figure 2 A sketch map to illustrate different samples in the fracture network.

\section{Results}

The correlation analysis between the 1D \& 2D intensity parameters and the 3D ones is summarized in Table 2. Sample orientations influence the correlations, and random sample orientations yield the 
Table 2: Correlations between 1D \& 2D intensity parameters and 3D intensity parameters

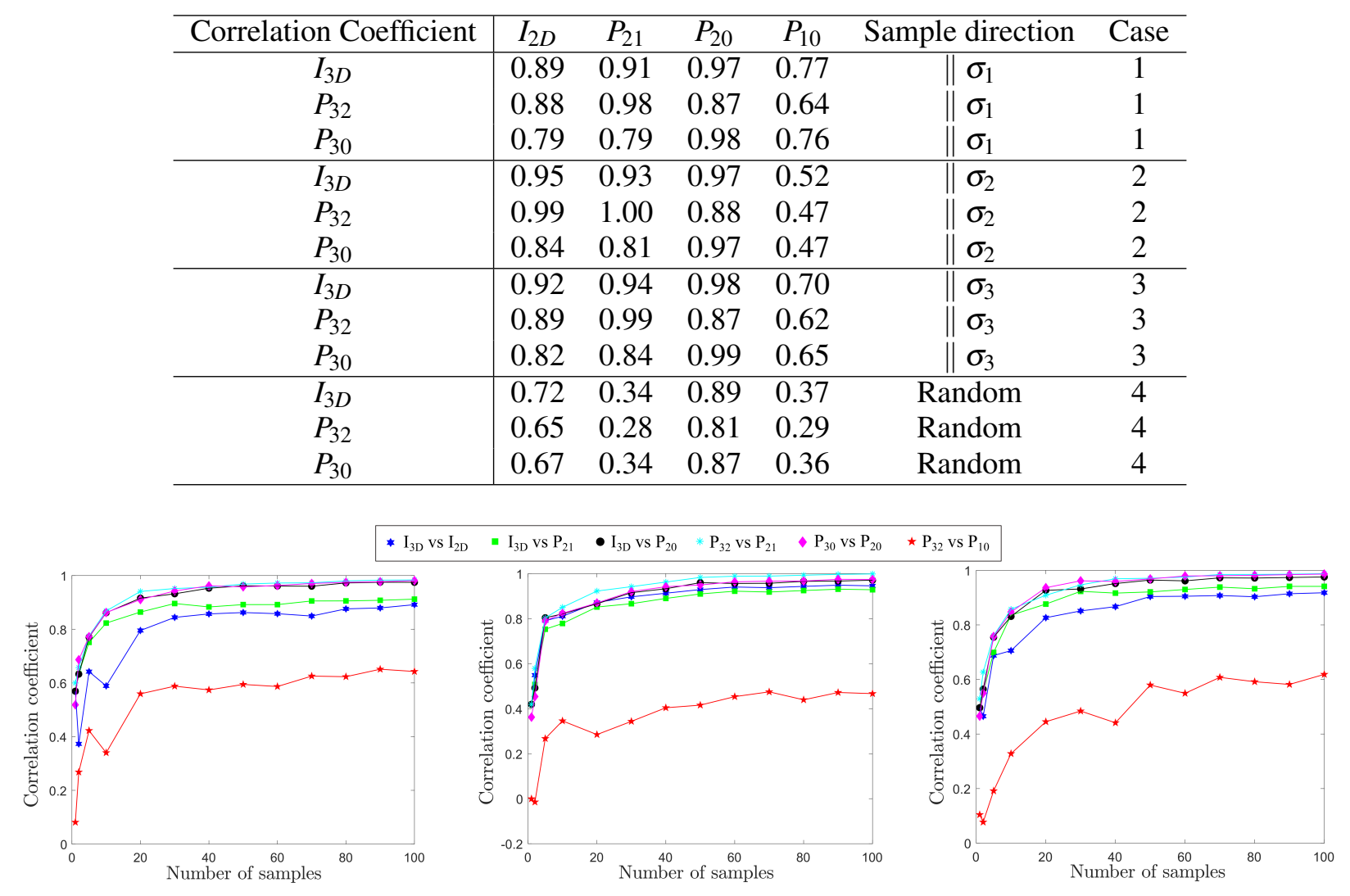

Figure 3 Variation of correlation coefficients with the numbers of samples to be averaged in the three cases (From left to right: Cases 1, 2 and 3).

poorest correlations between the lower and higher dimensionality intensity parameters. For the sample orientations parallel to principal stresses, $P_{10}$ doesn't correlate with any of the $3 \mathrm{D}$ intensity parameters, especially $P_{32}$. If samples are taken in the direction parallel to $\sigma_{2}$, the correlations are even worse. The 2D parameters, $I_{2 D}, P_{21}$ and $P_{20}$, all have good correlations with $I_{3 D}$ and $P_{32}$. $P_{30}$ has a stronger correlation with $P_{20}$, rather than with $I_{2 D}$ and $P_{21}$.

The results in Table 2 are averages of 100 samples. In practice, however, it is impossible to acquire enough samples for a meaningful correlation analysis. To investigate the minimum number of samples required for a good and stable correlation, we choose several pairs of parameters that have correlation coefficients larger than 0.9 . We also include $P_{32}$ and $P_{10}$, since this pair of parameters has been extensively studied by other researchers. The results of Case 1 - 3 are shown in Fig. 3. For all three cases, we find that the minimum number of samples required to have a good and robust correlation is about 20 . The correlations between small cube samples and original fracture networks are shown in Fig. 4. I $_{3 D}$
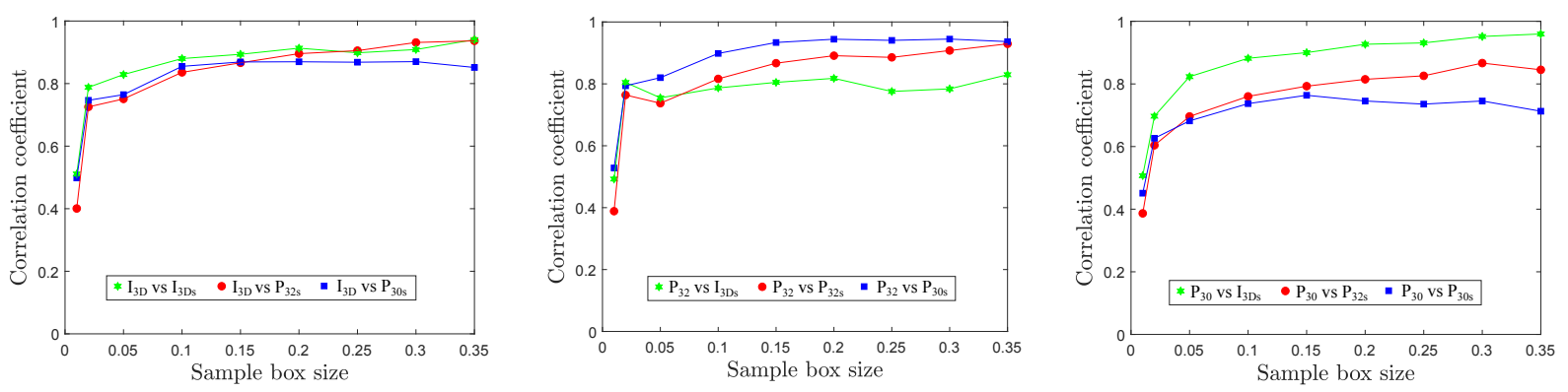

Figure 4 Variation of correlation coefficients with sizes of cube samples.

correlates well with all three 3D intensity parameters, $I_{3 D s}, P_{32 s}$ and $P_{30 s}$. $P_{32}$ correlates with $P_{32 s}$ and 
$P_{30 s} . P_{30}$ correlates well only with $I_{3 D s}$. When the size of the cube sample is larger than 10 percent of the system size, the correlation coefficients are larger than 0.8 , which means that the samples capture most of the information about the underlying network.

We also investigate the minimum number of samples required to have a good and stable correlation. We choose three pairs of parameters as examples to find this minimum number. These pairs are are $I_{3 D}$ and $P_{30 s}$ (Case 1), $P_{32}$ and $P_{32 s}$ (Case 2), and $P_{30}$ and $P_{30 s}$ (Case 3). The results are shown in Fig. 5. To achieve the correlation coefficient larger than 0.8 , the cube samples sizes must be 10 percent of the original system size, and the minimum number of samples about 60 .

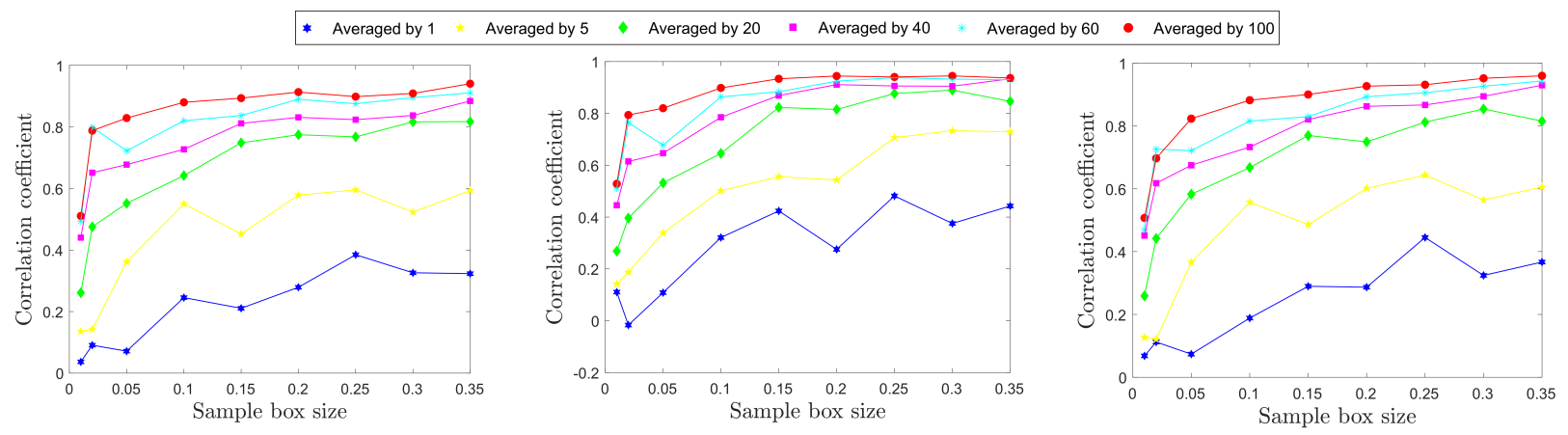

Figure 5 Variation of correlation coefficients for different sizes and numbers of the cube samples to be averaged (From left to right: $I_{3 D}$ vs $P_{30 s}, P_{32}$ vs $P_{32 s}, P_{30}$ vs $P_{30 s}$ ).

\section{Conclusions}

- Fracture sampling orientation impacts correlations between the 2D and 3D parameters. Samples parallel to the principal stresses yield better correlations.

- The 3D intensity parameters, $I_{3 D}, P_{32}$ and $P_{30}$, can be predicted from one or more $2 \mathrm{D}$ intensity parameters, e.g., $I_{2 D}, P_{21}$ or $P_{20}$. The minimum number of samples required for a good correlation is about 20 .

- The 1D logging measure, $P_{10}$, doesn't have a good correlation with the 3D intensity parameters, especially for the samples parallel to the intermediate principal stress $\sigma_{2}$.

- 3D intensity parameters can be predicted from one or more 3D intensity parameters of small cube samples. However, the cube sample sizes should be larger than 10 percent of the underlying fracture network, and the minimum number of cube samples should be larger than 60 .

\section{References}

Dershowitz, William S.and Herda, H.H. [1992] Interpretation of fracture spacing and intensity. The 33th U.S. Symposium on Rock Mechanics (USRMS), 757-766.

Dershowitz, W.S., Hermanson, J., Follin, S. and Mauldon, M. [2000] Fracture intensity measures in 1-D, 2-D, and 3-D at Aspo, Sweden. Pacific Rocks 2000: Rock around the Rim, 849-853.

Holland, M., Saxena, N. and Urai, J. [2009] Evolution of fractures in a highly dynamic thermal, hydraulic, and mechanical system - (II) Remote sensing fracture analysis, Jabal Shams, Oman Mountains. GeoArabia, 14, 163-194.

Wang, X. [2005] Stereological interpretation of rock fracture traces on borehole walls and other cylindrical surfaces. Doctoral dissertation, 113.

Zhu, W., Khirevich, S. and Patzek, T. [2018] Percolation Properties of Stochastic Fracture Networks in 2D and Outcrop Fracture Maps. In: 80th EAGE Conference and Exhibition 2018. EAGE Publications BV. 